\title{
Optimized Numerical Interface Conditions for Conjugate Heat Transfer
}

\author{
Marc-Paul Errera, Rocco Moretti, Yohann Bachelier \\ ONERA- The French Aerospace Lab \\ 29 Avenue de la Division Leclerc, 92320 Châtillon, France \\ marc.errera@onera.fr
}

\begin{abstract}
This paper presents the main numerical characteristics of conjugate heat transfer problems derived from a normal mode stability analysis based on the theory of Godunov-Ryabenkii. Two fundamental parameters are highlighted: a "numerical" Biot number controlling the stability process and an optimal coefficient that ensures unconditional stability. Examples in weak, moderate and strong thermal fluid-structure interaction illustrate the capabilities of the so-called optimal interface treatment.
\end{abstract}

Keywords: Coupling, Conjugate Heat Transfer, Stability, Interface, Optimal Schemes, Dirichlet, Robin.

\section{Introduction}

The term conjugate heat transfer (CHT) is used when the solid conduction and the fluid convection are analyzed simultaneously. CHT procedures are now commonly found in many real-word environments in which accurate heat transfer predictions are needed to design efficient cooling or heating systems. Coupled procedures are the most correct way to implement when accurate heat transfer predictions are needed. But quite often, arbitrary relaxation parameters are used to stabilize the numerical coupling procedure. Our goal in this paper is to present optimal interface conditions [1,2] derived from a stability analysis and to test them in weak, moderate and strong thermal fluid-structure interaction.

\section{Optimal Interface Treatment}

\subsection{Multiphysics Strategy}

The simulation of multiphysics problems is generally accomplished by partitioned staggered schemes [3,4]. Each system is treated by the discretization techniques with algorithms that are known to perform well individually. As a result, a stable fluid-solid solution will be sought by coupling a transient fluid solution with a steady solid state.

\subsection{Model Problem}

The nature of instabilities derived from a 1D model problem can give insight into the potential instabilities in 2D/3D computations. Thus, the behavior of interface conditions in CHT is often studied using a normal mode analysis applied to a model problem composed of two partitions with a shared interface.

\subsection{Dirichlet-Robin Interface Conditions}

A Robin condition is applied on the solid side to avoid destabilizing effects

$$
\hat{q}_{s}+\alpha_{f} \hat{T}_{s}=q_{f}+\alpha_{f} T_{f}
$$

The subscripts $f$ and $s$ denote the fluid and solid domain respectively and the $\left({ }^{\wedge}\right)$ notation indicates the sought values. $q$ is the interfacial heat flux $\left(\mathrm{W} \cdot \mathrm{m}^{-2}\right)$ and $T$ is the interface temperature. The general Robin condition (1) introduces the coupling parameter $\alpha_{f}\left(\mathrm{~W} \cdot \mathrm{m}^{-2} \cdot \mathrm{K}^{-1}\right)$ the choice of which directly influences the stability of the CHT process. On the fluid side, a Dirichlet condition is imposed $\left(\hat{T}_{f}=T_{s}\right)$. 


\subsection{Stability Analysis}

The Godunov-Ryabenkii (G-R) stability analysis is very similar to the standard Fourier stability [5]. A normal mode solution is applied to the discrete CHT model problem and leads to the following temporal amplification factor

$$
g\left(z, \alpha_{f}\right)=\frac{1}{\beta K_{s}+\alpha_{f}}\left[\kappa_{f}\left(\alpha_{f}, D_{f}\right)+\alpha_{f}-K_{f}\right]
$$

Where $K_{f}$ and $K_{s}$ are the fluid and solid conductances respectively $\left[\mathrm{W} . \mathrm{m}^{-2} \cdot \mathrm{K}^{-1}\right.$. We can thus define the mesh Biot number $B i^{(\Delta)}=K_{f} / K_{s}$. The parameter $\beta$ accounts for the external boundary condition. $\kappa_{f}$ is the "spatial" amplification factor that depends on the fluid mesh Fourier number $D_{f}$ (see [1] and [2] for more details).

\subsection{Numerical Biot Number}

The CHT process is stable in the sense of G-R if $\left|g\left(z, \alpha_{f}\right)\right|<1$. This condition applied to Eq.(2) leads, after introducing a normalized Fourier number $\bar{D}_{f}$, to the following thermal fluid-structure interaction :

- Weak interaction : $B i_{v}=B i^{(\Delta)}\left(1-\bar{D}_{f}\right) \leq 1$. If this condition holds, the "transient" thermal resistance of the fluid domain at the shared interface is greater than the resistance offered by the solid domain.

- Moderate interaction : $B i_{v}=B i^{(\Delta)}\left(1-\bar{D}_{f}\right) \geq 1$. This means that the solid thermal gradients are not negligible or that the thermal fluid conductance is larger than that of the solid.

- Strong interaction: $B i_{v}>>1$. This condition may be delicate and difficult for stability.

$B i_{v}$ is thus a fundamental number in the CHT process and can be regarded as the numerical Biot number.

\subsection{Optimal Coefficient}

The amplification factor is represented in Fig. 1 for weak $\left(B i_{v}=0.45\right)$, moderate $\left(B i_{v}=1.86\right)$ and strong $\left(B i_{v}=9.4\right)$ interaction. The shape of the three curves exhibits a transition when the curve switches and turns back.

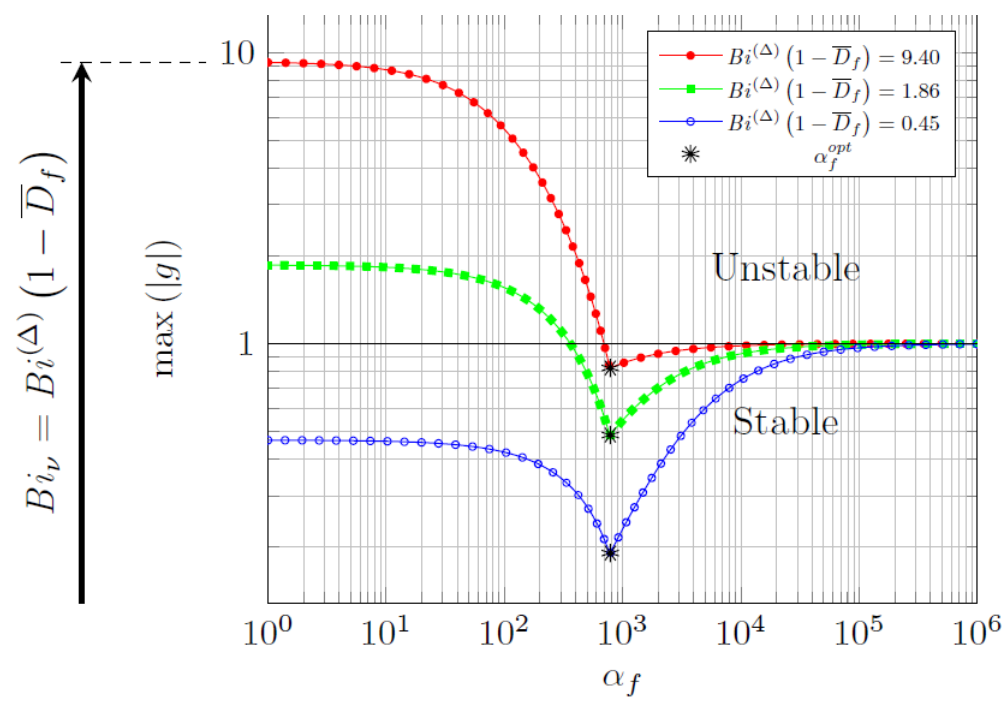

Fig. 1: Amplification factor of the coupled problem for weak/moderate/strong interaction. 
It can be shown [1], that this transition occurs at a unique and remarkable value $\alpha_{f}^{(o p t)}$ whose exact expression is given by

$$
\alpha_{f}^{(o p t)}=\frac{K_{f}}{1+\sqrt{1+2 D_{f}}}
$$

When the optimal value defined by (3) is employed, we obtain the best-case scenario with no additional computational effort. Note that $B i_{v}$ can also be defined as the $y$-intercept of the curves in Fig. 1 .

\section{CHT Computations}

\subsection{CHT Test Case}

CHT computations presented in this section are based on the Dirichlet-Robin interface condition. The coupled problem consists of convective heat transfer over, and conduction heat transfer within, a flat plate [6].

\subsection{Low Convergence and Oscillations}

Figure 2 displays the temperature profile at the leading edge of the flat plate. Two coupling coefficients have been used: $\alpha_{f}=0.61 * \alpha_{f}^{\text {opt }}$ and $\alpha_{f}=\alpha_{f}^{(o p t)}$.

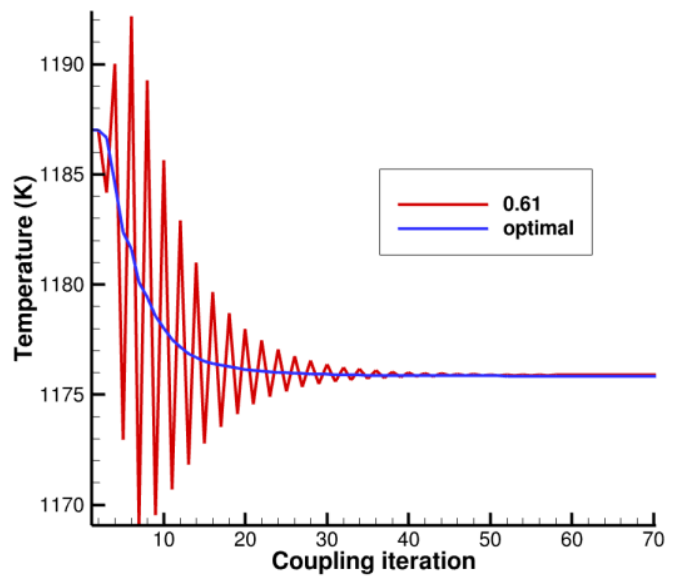

Fig. 2: Convergence history for two coupling coefficients.

It is clear from this figure that large oscillations occur during the initial coupling steps while a monotonic behavior is observed with the optimal coefficient. Finally, the oscillations die out after approximately 40 fluid-solid coupling periods. For $\alpha_{f}=\alpha_{f}^{(o p t)}$, a monotone and fast convergence is obtained as predictable by the model problem.

\subsection{Weak and Moderate Interaction}

Weak interaction is considered here by implementing a solid conductivity so as to obtain a numerical Biot number $B i_{v}=0.45$ (see Figure 1), i.e. lower than unity. The convergence history demonstrates that the coefficients $\alpha_{f}=0$ and $\alpha_{f}=\alpha_{f}^{(\text {opt })}$ provide roughly the same convergence history. Moderate interaction is then considered here by implementing a numerical Biot number $B i_{v}=1.86$ (see Figure 2). In this case, the computation for $\alpha_{f}=0$ is not unstable and convergence is reached. However, important oscillations of the interface temperature near the leading edge are observed initially. On the contrary, for $\alpha_{f}=\alpha_{f}^{(o p t)}$, the temperature behavior is oscillation-free. 


\subsection{Strong Interaction}

Strong interaction is considered (Biot number $B i_{v}=9.4$ ), i.e. much larger than unity. Figure 3 shows that, as expected, $\alpha_{f}=0$ leads to divergent computations at the $4^{\text {th }}$ coupling iteration. The other three coefficients result in stable computations. The fastest convergence is obtained for $\alpha_{f}=\alpha_{f}^{(o p t)}$.

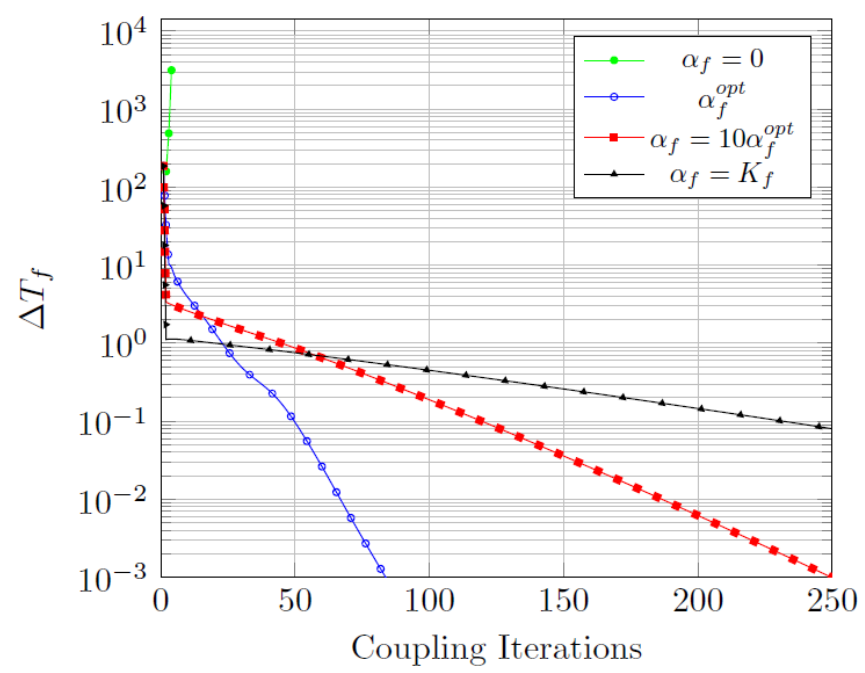

Fig. 3: Convergence history for four coupling coefficients and strong interaction $\left(B i_{v}=9.4\right)$.

\subsection{Comparison CFD/CHT}

At this point, it is of interest to stress that convergence is obtained for CHT after a number of iterations always smaller than the one needed for CFD even if these performances are pretty close (CPU time was decreased by $2 \%$ ).

\section{Conclusion}

We have shortly presented in this paper the main results of a stability analysis of a CHT problem based on the Dirichlet-Robin procedure. It has been seen that some key parameters are involved in the numerical behavior of the coupling process, such as the mesh Fourier number and a local transient Biot number. This enables us to set up an optimal procedure with no increase in computational effort. The resulting coupling method can be regarded as an adaptive procedure to always obtain the fastest rate of convergence and the best stability properties.

\section{References}

[1] M.-P. Errera and S. Chemin, "Optimal solutions of numerical interface conditions in fluid-structure thermal analysis," Journal of Computational Physics, vol. 245, pp. 431-455, 2013.

[2] M.-P. Errera and F. Duchaine, "Comparative study of coupling coefficients in Dirichlet-Robin procedure for fluidstructure aerothermal simulations," Journal of Computational Physics, vol. 312, pp. 218-234, 2016.

[3] C.A. Felippa and K.C. Park, "Staggered transient analysis procedures for coupled dynamic systems," Computer Met hods in Applied Mechanics and Engineering, vol. 24, pp. 61-1, 1980.

[4] S. Piperno, C. Farhat and B. Larrouturou, "Partitioned procedures for the transient solution of coupled aeroelastic problems," Computer Methods in Applied Mechanics and Engineering, vol. 124, pp. 79- 112, 1995.

[5] S. K. Godunov and V. S. Ryabenkii, "The theory of difference schemes. An introduction," North-Holland: Amsterdam, 1964.

[6] R. Moretti, M.-P. Errera, V. Couaillier, F. Feyel, "Stability, convergence and optimization of interface treatments in weak and strong thermal fluid-structure interaction," International Journal of Thermal Sciences, vol. 126, pp. 2337, 2018. 